Original Research Paper

\title{
Microscopic Study of the Lungs of Sprague-Dawley Rats from Various Ages of Postnatal and Aging Periods
}

\author{
${ }^{1}$ Ria Margiana, ${ }^{1}$ Isabella Kurnia Liem and ${ }^{2}$ Jeanne Adiwinata Pawitan \\ ${ }^{I}$ Department of Anatomy, Faculty of Medicine, Universitas Indonesia, Jakarta, Indonesia \\ ${ }^{2}$ Department of Histology, Faculty of Medicine, Universitas Indonesia, Jakarta, Indonesia
}

\section{Article history}

Received: 13-03-2015

Revised: $13-05-2015$

Accepted: 05-06-2015

Corresponding Author:

Ria Margiana

Department of Anatomy,

Faculty of Medicine,

Universitas Indonesia, Jakarta,

Indonesia

Email: riamargiana@yahoo.com
Abstract: Physiological changes in postnatal and aging lung are associated with a variety of microscopic changes in the lung, especially the alveolar lung tissue, both in the interstitial and epithelial component. Interstitial tissue of the lung will increase in thickness that is supposed to be due to changes in fiber composition, particularly collagen. However, the exact changes are still under debate and the underlying process is still unclear. The epithelial component that experiences changes is type II alveolar cells or pneumocyte II (surfactant producing cells). The ratio of pneumocyte II against pneumocyte I is expected to decline with age. This decrease will certainly affect their function in maintaining pulmonary surfactant supply. To maintain normal vital functions and synthesis of surfactant, lung tissue is also dependent on the availability of glucose because glucose is the fundamental building blocks of glycerol backbone of surfactant. In the aging process, accumulation of glycogen in the brain, skeletal muscle and kidney has been reported, so glycogen is supposed as a marker of cell aging (senescence cells). Nevertheless, studies on glycogen accumulation in lung tissue in aging have not been reported. This study aims to observe the microscopic changes in lung tissue, both in interstitial tissue and alveolar epithelial cell (pneumocyte II) in postnatal and aging Sprague-Dawley rats. This was an observational analytical study to determine the correlation between age and the amount of interstitial collagen fibers, the thickness of the interstitial tissue, glycoproteins and glycogen accumulation in the interstitial component, the ratio of pneumocyte II and I and accumulation of glycogen in pneumocyte II. Correlation tests were performed with Spearman correlation test $(\mathrm{p} \geq 0.05)$ using SPSS 17 for Windows. The study was conducted on Sprague-Dawley rats aged 2 days $(n=6), 4$ days $(n=6)$, 10 days $(n=6), 16$ days $(n=6), 3-4$ months $(n=6)$ and more than 12 months $(n=6)$. Lung tissue was cut with a thickness of $5 \mu \mathrm{m}$ and stained with Mason's trichrome to visualize collagen and with periodic acid Schiff to visualize glycoproteins and glycogen. There was a low correlation between age and proportion of interstitial tissue $(\mathrm{r}=0.291)$, the accumulation of glycoproteins in the interstitial $(r=0.300)$ and accumulation of glycogen in pneumocyte II $(r=266)$. There was a moderate correlation between age and the amount of interstitial collagen fibers $(r=0.687)$, but there was no correlation between age and pneumocyte II/I ratio $(\mathrm{r}=0.123)$. With increasing age, the interstitial lung tissue would increase in thickness, collagen fibers and glycoproteins and glycogen accumulation. While in the lung epithelium, no evidence of decline in the ratio of pneumocyte II/I, but there is an increasing accumulation of glycogen in pneumocyte II.

Keywords: Lung Parenchyma, Pulmonary Postnatal, Lung Aging, Lung Morphology 


\section{Introduction}

Aging of the lungs is a normal process that occurs since birth (Pinkerton and Green, 2004). Aging process causes changes in the physiology, metabolism and immunity of the lungs. As age increases, the airway and lung tissues become less elastic and more rigid. Lung vital capacity decreases to $35 \%$ at age 70 . In addition, there is a decrease in blood oxygen supply and the activity of alveolar macrophages. Hence in old age, the lungs respond less to physiological stress and diseases and are more prone to injuries such as pneumonitis, bronchitis, emphysema, Chronic Obstructive Pulmonary Disease (COPD), asthma and other lung disorders (Tortora, 1999; Defeo and Carson, 2009). In experimental animal observations, minimum emphysema and signs of fibrosis associated with focal pneumonitis were found in the lungs of aging canines, just like in humans. The tendency of pulmonary edema was discovered in mice and rats.

It seems that the changes in the physiology of lungs are not only related to the changes in the anatomy of the chest region (thorax), but are also associated with the changes in the morphology of lung parenchyma, especially the alveolar tissues of the lungs as the most important part of lung physiology in gas exchange process (oxygen and carbon dioxide). As age increases, lung elastic tissues are replaced by collagen, resulting in the narrowing of airway size. The diameter decline in the airway size will increase the volume of alveoli's residual air during expiration. In addition, the reduction in alveolar surface area, changes in alveolar structure and collagen deposition in the alveolar wall will cause an increase in alveolar-arterial oxygen gradient (Tortora, 1999). In the lungs of aging mice and rats, pulmonary hyperinflation with significant widening of alveolar air space and the size increase of interalveolar pores were discovered, which allegedly facilitate the occurrence of edema. The mechanism underlying these changes is unclear, but is thought to be caused by the damage to the pulmonary parenchyma due to aging, the rapid enlargement of the lungs after birth (postnatal), or the degenerative process during the aging process. Although it is still debated, the decline in lung elasticity is suspected due to the decrease of elastic fibers and the decrease of collagen fibers of the pulmonary interstitial tissues (Pinkerton and Green, 2004).

Through morphometric analysis of pulmonary interstitial matrix, it is known that the volume of the interstitial matrix also increases in aging. However, this does not appear to be caused by pulmonary edema, but is thought to be caused by the continuous increase of the collagen fibers in the pulmonary interstitial tissue. As reported in the kidneys (Dong et al., 2007; Zhou et al., 2004; Verbeke et al., 1997), accumulation of collagen fibers may be associated with the increase of glycation products, namely advanced glycation end products
(AGEs) in pulmonary interstitial, which lead to fibrosis. Because there is no satisfactory explanation of the underlying mechanism (Pinkerton and Green, 2004), interpret the continuous rise of collagen fibers as agerelated excess of collagen or fibrosis. The accumulation of AGEs in interstitial matrix is also believed to cause the thickness changes of basement membranes (Rahbar and Figarola, 2003). Alveolar wall consists of cellular and non cellular components that plays an important roles in maintaining optimum lung function. The most important cells are type I and II pneumocytes (Fawcett, 1994).

This study aimed to determine the microscopic changes of the lungs of Sprague-Dawley rats of various ages of postnatal and aging periods, in term of amount of interstitial tissue, amount of collagen fibers, glycoprotein and glycogen in interstitial tissue, ratio of type II pneumocytes to type I pneumocytes and glycogen accumulation in type II pneumocytes.

\section{Materials and Methods}

This study was an observational analytical laboratory study. The study was conducted from January to June 2011 in the Animal Laboratory for Histology Research, Laboratory of Histology and Laboratory of Anatomy of the Faculty of Medicine, Universitas Indonesia, Jakarta. This study used Sprague-Dawley rats aged 2 days, 4 days, 16 days and 3-4 months obtained from the Experimental Animal Division of the Research and Development of the Department of Health of the Republic of Indonesia. Sprague-Dawley rats aged 10 days were procured from the Animal Laboratory for Histology Research, while rats aged more than 12 months were from the Animal Laboratory of Bogor Agricultural University.

There were 6 groups, each group consisted of 8 rats. Rats' body weights were calculated by weighing using two kinds of tools. For neonates aged 2 days, 4 days and 10 days, mass scale was used, whereas for neonates aged 16 days, 3-4 months and more than 12 months analogue scale was used.

Rats were sacrificed at ages 2, 4, 10 and 16 days of the postnatal period, ages three to four months and ages more or equal to 12 months. Neonates were anesthetized using hypothermia method; young adult and mature adult rats were anesthetized with $10 \mathrm{mg} \mathrm{kg}{ }^{-1}$ body weight ketamine and $10 \mathrm{mg} \mathrm{kg}^{-1}$ body weight xylazine.

To get the exact hypothermia method duration for neonatal rats, a preliminary study was conducted. Rats were inserted into a room filled with shaved ice and then the time until the rats fell asleep was calculated with a stop watch and recorded.

After the rats fell asleep due to anesthesia, they were placed on the operating table for experimental animals. Thoracotomy was conducted by skin incision and muscle tissue in the middle of the chest and rib cartilage (in costosternal joint) were cut open. After the chest cavity had been opened, the left ventricle of the heart was identified and inserted with a needle of a syringe pump 
for intravital fixation. Fixative fluid flowed from the glass beaker into the pump engine to the needle fixed in the left ventricle of the heart. This process took up around $30 \mathrm{~min}$. The organs that were taken were sliced into 2-3 mm thickness using lancet with a cross-section cut and then were placed in a container of formol-saline. They were left in room temperature for $24 \mathrm{~h}$ and 5 micron microscopic slides were made. The slides were stained by Masson's Trichrome and PAS advanced staining.

Result of Mason-trichrome staining: Collagen would be stained purplish blue, while that of muscle tissues and cornified epithelial keratin layer would be stained red and nuclei would be stained blue to black.

Result of PAS staining: Glycogen and other carbohydrates that were reactive to Periodic Acid Schiff would be stained magenta and nuclei would be stained blue.

\section{Data Collection}

From each lung microscopic slide of each rat group, 10 random high-power fields were taken in the alveolar region. The fields were photographed with Vimicro photo camera. All the microphotography results were stored in computer files in bmp format. Image analysis to obtain data was done by Optilab Raster Image software.

Data collected were: Thickness of interstitial tissue and amount of collagen fibers (from Mason trichrome staining) and amount glycoprotein and glycogen (from PAS staining).

The data were calculated and recorded as follows:

- The thickness of the interstitial tissue was calculated by measuring the width of interstitial tissues compared with the width of the field of view of the lungs

- The number/amount of collagen fibers was measured semi-quantitatively based on the width of distribution with the following conditions:

- $\quad+1$ : Narrowly distributed

- $\quad+2$ : Moderately distributed

- +3: Widely distributed

- Glycoprotein and glycogen accumulation in the interstitial tissues was measured semi-quantitatively based on the width of distribution and colour intensity with the following conditions:

- $\quad+1$ : Narrowly distributed and low intensity

- $\quad+2$ : Moderately distributed and medium intensity

- $\quad+3$ : Widely distributed and strong intensity

- $\quad$ Ratio of type II to type I pneumocytes was obtained by calculating the number of type II and type I pneumocytes per one hundred pneumocytes

- Glycogen accumulation in type II pneumocytes was measured semi-quantitatively based on PAS colour intensity on 100 type II pneumocytes with the following conditions:
- $0=$ no color

- $\quad+1=$ weak intensity

- $\quad+2=$ moderate intensity

- $\quad+3=$ strong intensity

\section{Statistical Analysis}

The data were analyzed with SPSS version 17. Statistical analysis began with data normality test. Normal data obtained would be analyzed using Pearson correlation test and not normal data would be analyzed using Spearman correlation test with $\mathrm{p}=0.05$.

\section{Results}

Research sample characteristics, measurement results, observations and calculations and the results of analysis concerning relationship between ages and various variables can be seen below.

\section{Weight}

The number of total samples for the six groups was 36 rats. The average weight and Standard Deviation (SD) of each group can be seen in Table 1 .

\section{Duration of Hypothermia for Anesthesia}

Hypothermia anesthesia in rats aged 2, 4 and 10 days and the results can be seen in Table 2 .

The result of the time duration calculation was reported as a preliminary study at the meeting of the Indonesian Anatomist Association (IAA) in 2010. Spearman correlation test showed a strong correlation between the duration of hypothermia and age $(r=0.896$, $\mathrm{p} \geq 0.05)$ and body weight of rats $(\mathrm{r}=0.827, \mathrm{p} \geq 0.05)$.

\section{Interstitial Tissue Proportion}

Measurement of the average interstitial tissue proportion was done by measuring the width of interstitial tissues compared with the width of the field of view of the lungs. The complete result can be seen in Table 3. The method to measure the proportion of pulmonary interstitial tissues can be seen in Fig. 1.

\section{Collagen Fiber in Interstitial Tissue}

Distribution of collagen fiber in lung interstitial tissue (semiquantitative) can be seen in Fig. 2.

\section{Glycoprotein in Interstitial Tissue}

Glycoprotein Analysis in Interstitial Tissue can be seen in Fig. 3.

\section{Ratio of Type II to Type I Pneumocyte}

The calculation result of type II/I pneumocyte ratios can be seen in Table 4, whereas the ratio calculation image of type II/I pneumocytes can be seen in Fig. 4 . 
Ria Margiana et al. / OnLine Journal of Biological Sciences 2015, 15 (2): 74.82 DOI: $10.3844 /$ ojbsci.2015.74.82

\begin{tabular}{ll} 
Table 1. Rat's body weight & \\
\hline Subject & Mean of Body Weight (gram) \\
\hline 2 days old & $6.67 \pm 0.53$ \\
4 days old & $10.24 \pm 0.30$ \\
10 days old & $14.38 \pm 0.30$ \\
16 days old & $28.81 \pm 1.27$ \\
3-4 months old & $278 \pm 17.76$ \\
$>12$ months old & $264 \pm 22.38$ \\
\hline
\end{tabular}

Table 2. Duration Required for hypothermi anesthesia

\begin{tabular}{ll}
\hline Sample & Mean of Duration (minute) \\
\hline 2 days old & $4.8 \pm 0.13$ \\
4 days old & $6.9 \pm 0.41$ \\
10 days old & $10 \pm 0.82$ \\
\hline
\end{tabular}

Table 3. Proportion of interstitial tissue width in one

\begin{tabular}{ll}
\hline Subject & $\begin{array}{l}\text { Mean of Proportion of Interstitial } \\
\text { Tissue Width in One High- } \\
\text { Power Field }(\mu \mathrm{m})\end{array}$ \\
\hline 2 days old & $0.17 \pm 0.01$ \\
4 days old & $0.19 \pm 0.01$ \\
10 days old & $0.18 \pm 0.01$ \\
16 days old & $0.19 \pm 0.01$ \\
3-4 months old & $0.29 \pm 0.01$ \\
$>12$ months old & $0.30 \pm 0.01$ \\
\hline
\end{tabular}

Table 4. Ratio of type II to type I pneumocytes in 100

\begin{tabular}{ll}
\hline Subject & $\begin{array}{l}\text { Ratio of Type II to Type } \\
\text { I Pneumocytes in } 100 \\
\text { Pneumocytes }\end{array}$ \\
\hline 2 days old & $0.34 \pm 0.033$ \\
10ys old & $0.31 \pm 0.022$ \\
16 days old & $0.31 \pm 0.040$ \\
3-4 months old & $0.35 \pm 0.033$ \\
$>12$ months old & $0.36 \pm 0.032$ \\
\hline
\end{tabular}

From Table 4, the ratio of type II to type I pneumocytes between ages did not differ significantly, they were ranging from $0.31 \pm 0.022$ to $0.36 \pm 0.032$.

Glycoprotein accumulation in type II pneumocytes. Glycoprotein accumulation in type II pneumocytes can be found in Fig. 4.

\section{Result of Correlation Test of Dependent and Independent Variables}

In normality test, all of the variables were not normal, hence all of the variables must be analysed with Spearman correlation test. There was a weak correlation between age and interstitial tissue proportion $(r=0,29)$, between age and glycoprotein in interstitial tissue $(r=0,300)$ and between age and glycoprotein content in type II pneumocytes $(\mathrm{r}=$ $0,266)$. There was a medium correlation between age and collagen $(r=0,687)$, but there was no correlation between age and the ratio of type II/Type I pneumocytes $(r=0,123)$.

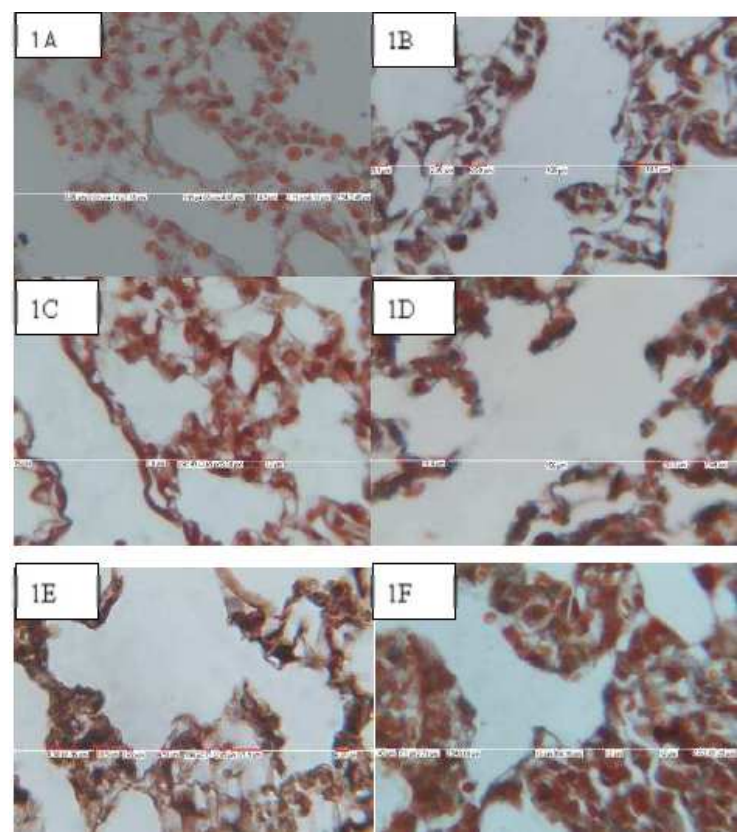

Fig. 1. Measurement of lung interstitial tissue proportion. 1.A. Day-2. interstitial tissue proportion 0.17; 1.B. Day-4. interstitial tissue proportion 0.19; 1.C. Day-10. interstitial tissue proportion 0.18; 1.D. Day-16. interstitial tissue proportion 0.19 ; 1.E. 3-4 months. interstitial tissue proportion $0.29 ; 1 . F .>12$ months. interstitial tissue proportion 0.30

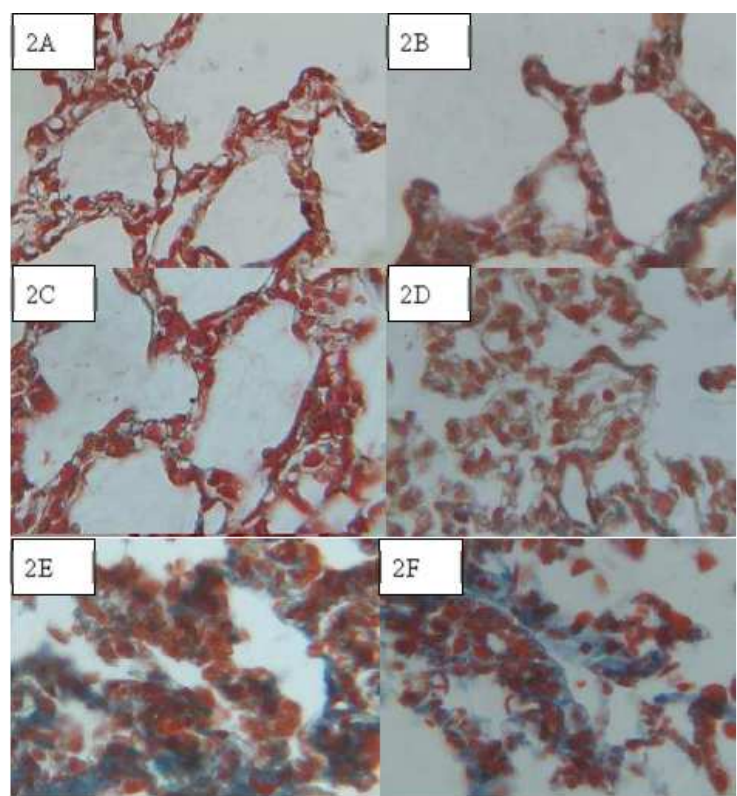

Fig. 2. Distribution of collagen fiber in lung interstitial tissue. 2A. 2 days old. Collagen distribution: +1 ; 2.B. 4 days old. Collagen distribution: +1 ; 2.C. 10 days old. Collagen distribution: +1 ; 2.D. 16 days old. Collagen distribution: +2 ; 2.E. 3-4 months old. Collagen distribution: +3 ; 2.F. $>12$ months old. Collagen distribution: +3 

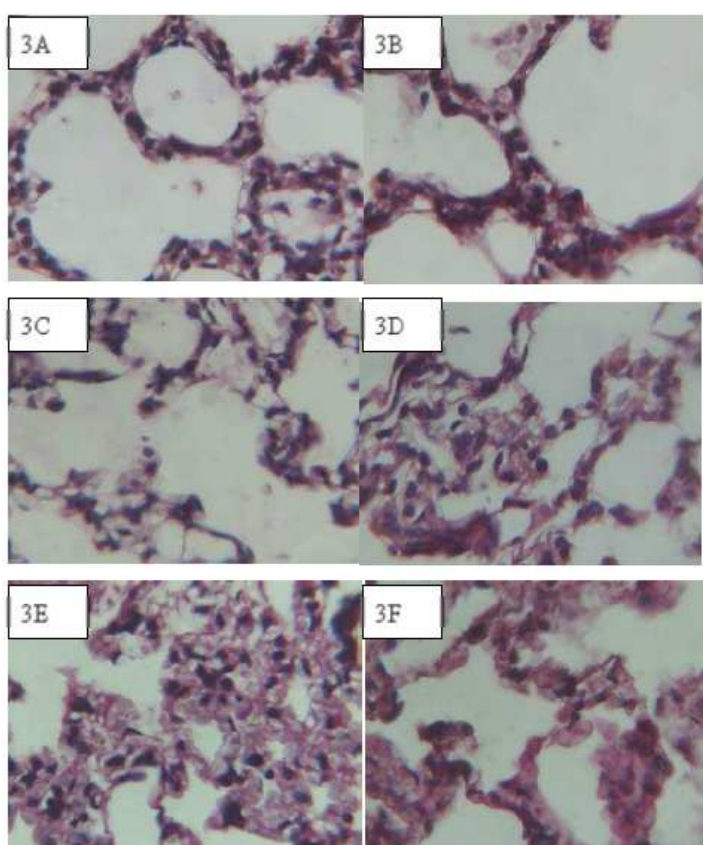

Fig. 3. Glycoprotein Analysis in Interstitial Tissue. 3. A. 2 days old. Glycoprotein Analysis in Interstitial Tissue: +1 ; 3.B. 4 days old. Glycoprotein Analysis in Interstitial Tissue: +2; 3.C. 10 days old. Glycoprotein Analysis in Interstitial Tissue: +1; 3.D. 16 days old. Glycoprotein Analysis in Interstitial Tissues +2; 3-4 months old. Glycoprotein Analysis in Interstitial Tissue: +3; 3.F. $>12$ months. Glycoprotein Analysis in Interstitial Tissue: +4
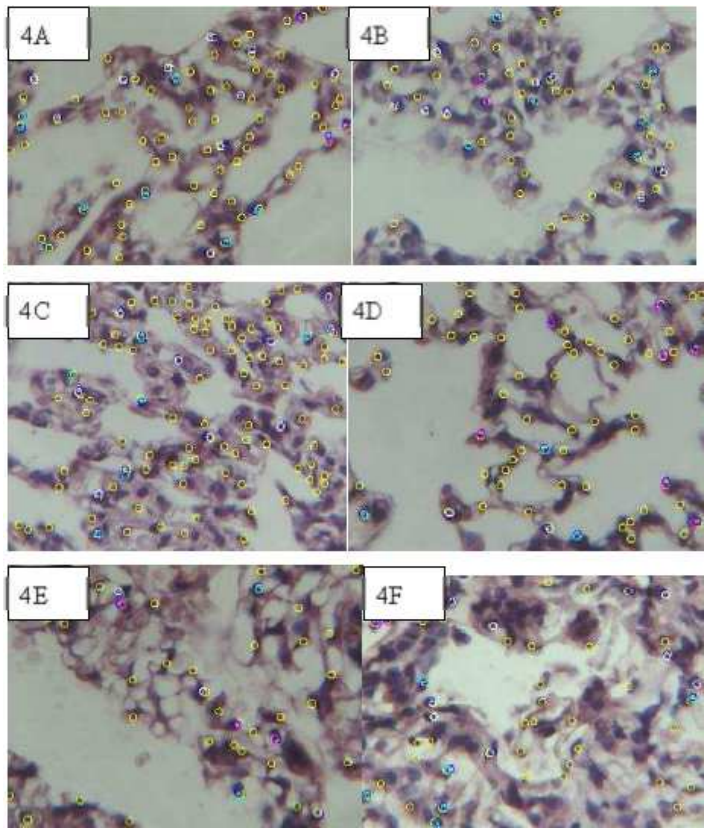

Fig 4. Glycoprotein Accumulation in Type II Pneumocytes. 4.A. 2 days old. Glycoprotein accumulation in type II pneumocytes: $+1=4,+2=8,+3=10$; 4 .B. 4 days old. Glycoprotein accumulation in type II pneumocytes: $+1=$
$2,+2=8,+3=6 ; 4$.C. 10 days old. Glycoprotein accumulation in type II pneumocytes: $+1=1,+2=6,+3$ $=9 ; 4 . D .16$ days old. Glycoprotein accumulation in type II pneumocytes: $+1=8,+2=3,+3=2$; 4 .E. $3-4$ months old. Glycoprotein accumulation in type II pneumocytes: $+1=3,+2=2,+3=4 ; 4$.F. $>12$ months old. Glycoprotein accumulation in type II pneumocytes: $0=2,+1=6,+2=8,+3=1$

\section{Discussions}

Changes in the Lung Interstitial Tissue of the Sprague-Dawley Rats of Various Ages at Postnatal and Aging Period

Many studies and observations in developing and aging lung tissues have been implemented, but none has been able to provide thorough description on the morphology changes and satisfactory explanation on the relationship with physiological changes. We have conducted a morphological observation on the pulmonary parenchyma and stroma of Sprague-Dawley rats at neonatal $(2,4,10$ and 16 days), young adult (3-4 months) and mature adult (over 12 months) periods to get an overview of the morphological changes of pulmonary parenchyma and stroma at postnatal and aging periods more thoroughly. The lung tissue staining techniques used were Masson's Trichrome staining protocol to detect collagen and Periodic Acid Schiff (PAS) to detect glycoprotein and glycogen. Although in this study glycation products (AGEs) cannot be detected directly, by determining the changes in lung tissue morphology and detecting glycoprotein in the interstitial tissues through both staining processes, we expect that this study can provide a clearer picture on the changes of aging lung tissue microscopic images and the mechanism underlying them.

In this study, there were three variables of pulmonary interstitial tissues studied, i.e., the thickness of the interstitial tissues obtained from interstitial tissue proportion, the amount of collagen fibers and glycoprotein and glycogen accumulation. Although they did not show a strong correlation, the results showed that along with the increase of age, there was a tendency of interstitial tissue increase, collagen fiber rise and glycoprotein and glycogen accumulation in the interstitial tissues.

The interstitial tissue proportions between the neonatal periods were not significantly different, which was about 0.17 to 0.19 . However, in young and mature adults, proportion increases were i.e., to 0.29 and 0.30 . According to (Pinkerton and Green 2004), during development, more rigid interstitial tissues increases, due to the formation of glycosaminoglycans, which are important to induce airway formation and terminal sac formation. During postnatal period, interstitial tissues begin to soften because their function is being replaced 
by connective tissue fibers. Pinkerton and Green reported an increase in the number of interstitial tissue components at the age of 5, 14 and 26 months. Although in this study the calculated item was the interstitial tissue proportion, the research results of our study supports the research results reported by Pinkerton and Green. There was a weak correlation between interstitial tissue and age, which was associated with an increase at older age, albeit small. Therefore, a study in older rats is necessary.

During the saccular phase of lung development (E21$\mathrm{P} 4$ ), which is on the second day, the interstitial tissue proportion is smaller than the initial alveolarization phase (P4-P14), which is on the fourth day. This is possibly related to an increase in interstitial fibroblast proliferation. However, on the tenth day, the interstitial tissue proportion declines. At the end of alveolarization/remodeling phase (P14 to adulthood), which is on the sixteenth day and 3-4 months of age, the interstitial tissue proportion starts to rise again, in accordance with the development of age (Pinkerton and Green, 2004).

Pinkerton and Green reported that the ratios of collagen and elastin changed significantly from 3-5 at 46 months of age to 10 at the age of 20-24 months. This study showed the tendency of increased collagen fibers along with age. However, further research is needed to compare the number of elastic fibers. Collagen stained with Masson's Trichrome staining technique cannot differentiate the different types of collagen in the lungs. It is known that the majority of interstitial collagen is type I collagen, while the collagen resided in the basement membrane is generally type IV collagen (Pinkerton and Green, 2004). In this study, there was amoderate correlation between the width of collagen fibers and age; although the increase was small, but it was consistent with the increase of age.

Correlation test result between the interstitial tissue proportion and collagen fibers showed weak correlation $(\mathrm{r}=0.211)$. This fact indicates the possibility of interstitial tissue thickening that may be caused by an increase in the number of collagen fibers. However, to ensure this, more study needs to be implemented on the components of other interstitial tissues, such as elastic fibers. Glycoprotein in interstitial tissues is assumed to rise because in one of aging theories AGE formation reaction occurs, which is caused by glycogen that can react with various proteins in lung tissues. Moreover collagen is also a glycoprotein. Collagen produced from this reaction is less favorable because it is more rigid than the supposed collagen (Dong et al., 2007; Zhou et al., 2004; Verbeke et al., 1997). In this study, there was a weak correlation between the glycoprotein in the interstitial tissues and age. Rats used in this study were 2 days old, in accordance with the saccular phase; 4 and 10 days old, in accordance with the initial alveolarization phase; and 16 days old, in accordance with the final alveolarization or remodeling phase. The correlation between interstitial glycoprotein and age is probably because during the alveolarization phase, the interstitial fibroblast proliferation peak occurs, with glycogen as the energy source (Pinkerton and Green, 2004). In this study, glycogen increased with age although the correlation was weak. This fact is probably due to the reaction of AGE formation; however, more study needs to be done using AGE special marker. Glycoprotein in this study was detected by PAS staining; PAS staining has the disadvantage of detecting not only glycoprotein but also all the components containing aldehyde group such as pure glycogen, mucus and even collagen (Pinkerton and Green, 2004).

In the skin, the glycation process affects the characteristics of collagen. The accumulation of glycated collagen products (by Maillard reaction) causes the collagen to become more rigid and brittle (Verzijl et al., 2000). Glycation is reported to cause aggregation of collagen monomers into the constituent fibers of extracellular matrix (Guitton et al., 1981). Additionally, glycation can also influence extracellular matrix itself and modulate the characteristics of some resident cells, which normally reside in the lungs, such as fibroblasts in interstitial tissues, pneumocytes and others. Cell characteristics that experience changes are the process of migration, growth, proliferation, differentiation and expression of genes. Phenomena mentioned above can explain how glycation in extracellular matrix affects the characteristics of a cell. (Singh et al., 2001).

Correlation test result between the number of collagen fibers in the interstitial tissues and glycoprotein glycogen accumulation in the interstitial tissues showed weak correlation $(r=0.283)$; this implies that one of the causes of increased interstitial tissues is the increase in glycogen and interstitial glycogen. The underlying booster of glycoprotein and glycogen quantity in interstitial according to some literature is AGE production increase, like in other tissue such as in the skin etc. (Dong et al., 2007; Zhou et al., 2004; Verbeke et al., 1997). Therefore, it is necessary to do further study using AGE special marker in the lung parenchyme.

The increased thickness of the interstitial tissues and collagen fibers in the aging lungs indicates that some pathological conditions occur more easily, e.g., interstitial fibrotic pulmonary disease, COPD, pulmonary edema and others.

\section{Changes in Lung Epithelial Cells of Sprague- Dawley Rats of Various Ages at Postnatal and Aging Period}

To maintain the normal and vital functions of surfactant synthesis, lung tissues depend on the high availability of glucose because glucose is the forming component of surfactant glycerol backbone (Ross and Post, 2004). The glucose compliance is derived mostly from the constant 
supply of external glucose and slightly from the glucose deposit (glycogen) in the lung tissues (Atwal and Bray, 1981). In physiological conditions, intracellular glycogen accumulation/buildup will not appear. However, in certain circumstances, such as in the precursors of type II pneumocytes that have not been differentiated (in the fetal period) or during the proliferation of type II pneumocytes, for example at the time to differentiate into type I pneumocytes, intracellular glycogen accumulation will occur (McGowan and Snyder, 2004). In pathological conditions, for instance pulmonary edema induced by 3Methylindole (experiment on goat), Atwal and Bray (1981) also found an accumulation of glycogen in type II pneumocytes (Atwal and Bray, 1981). They believe that this glycogen accumulation has negative impact on the production of surfactants, with rationale that glycogen accumulation is the manifestation of the breakdown failure from glycogen to glucose. The failure of glucose formation that is essential for the synthesis of phospholipids ultimately leads to the reduced production of surfactants. Glycogen accumulation reportedly also occurs in aging brains, skeletal muscles and kidneys; (Mann et al., 1987; Manchester et al., 1996; Kang et al., 2005) hence, there are opinions that glycogen is the marker of aging cells (senescent cells). Lin et al. (2001) However, researches on glycogen accumulation in aging lung tissues have not been reported.

The lung epithelial cells observed in this study were the ratio of type II to type I pneumocytes and glycogen accumulation/build up in type II pneumocytes. Although it has been reported previously, the ratio of type II pneumocytes to type I pneumocytes was recalculated because the data was necessary to assess the specific ratios of the defined age groups in this study; in addition, the data on glycogen accumulation in type II pneumocytes should also be preceded by the calculation of the quantity of type II pneumocytes. However, no study that observed the presence/absence of glycogen accumulation in type II pneumocytes has been reported. We implemented the study because the data that directly observe the relationship between age (mainly related to the development and aging) and glycogen accumulation are not available yet.

Pulmonary alveolar epithelial components experiencing dramatic changes during postnatal development also undergo changing during the aging process, particularly type II alveolar cells or type II pneumocytes (surfactant-producing cells) (Guthman et al., 1998). In rats, the ratios of type II to type I pneumocytes (type I alveolar cells) decrease along with the increase of age; from 1.8 at the age of 6 weeks to 1.0 at the age of 26 weeks. The cause of this ratio decline is not yet known, but it can contribute to the deviation of secretory response of type II pneumocytes of older rats due to wider surface area that has to be served by type II pneumocytes compared with younger rats (Pinkerton and Green, 2004). Thus, as age increases, the sustainability of surfactant synthesis by type II pneumocytes to ensure sufficient surfactants becomes an increasingly vital thing in keeping normal lung functions and maintaining pulmonary resistance against exposure to hazardous substances (Atwal and Bray, 1981).

The results of this study showed that type II/I pneumocyte ratio did not correlate with age. In this study, neonates aged 2 days old show a ratio of 0.34 , which then decreased at the age of 4 days and 10 days old. The cause of the decline in the postnatal age is unknown, but perhaps it is related to the pneumocyte function as surfactant producer. At the age of 2 days, surfactant production is still required; therefore the number of type II pneumocytes in this study was more compared to that of 4 days and 10 days old. For ages of 16 days and 3-4 months, type II/I pneumocyte ratios tended to be stable; at the age of 16 days, rats are considered to be able to use their lungs maximally just like young adult rats. At the age of over 12 months, type II/I pneumocyte ratio in this study started to slightly decline; this might be due to the widening surface area to be maintained and type II pneumocytes as stem cells might differentiate into type I pneumocytes. It is known that lung epithelial cells that play an important role in the gas exchange system are type I pneumocytes.1 (Pinkerton and Green, 2004). In this study, during the saccular phase of lung development (E21-P4), which was on the second day, type II/I pneumocyte ratio was higher than the initial alveolarization phase (P4-P14), which was on the $4^{\text {th }}$ and $10^{\text {th }}$ day, when type II/I pneumocyte ratios were stable. At the end of final alveolarization/remodeling phase (P14 to adulthood), which was on the 16th day and 3-4 months old, type II/I pneumocyte ratios were on the rise again. At mature adults, type II/I pneumocyte ratios slightly decreased. Our results was consistent with Pinkerton study that showed that type II and I pneumocyte ratios declined at old age, i.e., at the age of 5 months with a ratio of 1.8 and 26 months with a ratio of 1.0 (Pinkerton and Green, 2004).

The content of glycogen in type II pneumocytes after birth is generally plummeted drastically down to $10 \%$ compared to just before birth. 1 We suspect there will be an increase in glycogen content in aging period, but it turned out to be a weak correlation between age and glycogen accumulation in type II pneumocytes. Thus, further study to compare the conditions before birth, after birth, until aging periods is needed. 


\section{Conclusion}

In the lung tissues of Sprague-Dawley rats of various ages of postnatal and aging periods, there were increased interstitial tissues, increased amount of collagen fibers in the interstitial tissues and glycoprotein and glycogen accumulation in the interstitial tissues and glycogen accumulation in type II pneumocytes. Further, there was no decline in the ratios of type II to type I pneumocytes. The ratios of type II pneumocytes to type I pneumocytes are possibly related to development stages of the lungs instead of age.

\section{Acknowledgement}

This study was supported by Department Anatomy and Histology Faculty of Medicine Universitas Indonesia.

\section{Funding Information}

The authors have no support or funding to report.

\section{Author's Contributions}

Ria Margiana: Laboratory activities, developing ideas, writing the paper publication.

Jeanne Adiwinata Adiwinata: Laboratory consultant, improving the idea, prove reading the paper publication.

Isabella Kurnia Liem: Laboratory consultant, improving the idea, prove reading the paper publication.

\section{Ethics}

This study has been approved by Research Ethical Committee Faculty of Medicine Universitas Indonesia.

\section{References}

Atwal, O.S. and T.M. Bray, 1981. Glycogen accumulation in alveolar type II cells in 3methylindole-induced pulmonary edema in goats. Am. J. Pathol., 105: 255-262. PMID: 6274198

Defeo, D. and S.S. Carson, 2009. Pulmonary Issues in the Elderly. In: Reichel's Care of the Elderly: Clinical Aspects of Aging, Arenson, C., J. Busby-Whitehead, K. Brummel-Smith, J.G. O'Brien and M.H. Palmer et al. (Eds.), Cambridge University Press, Cambridge, ISBN-10: 1139476408, pp: 221-230.

Dong, Y.H., L.X. Zhu, X.J. Mei and L. Man, 2007. Effects of advanced glycation end products on renal fibrosis and oxidative stress in cultured NRK-49 F cells. Chin. Med. J., 120: 787-793. PMID: 17531120
Fawcett, D.W., 1994. Respiratory system. In: Bloom and Fawcett: A Textbook of Histology, Fawcett, D.W., (Ed.), Chapman and Hall, New York, pp: 704-727.

Guitton, J.D., A. Le Pape, P.Y. Sizaret and J.P. Muh, 1981. Effects of in vitro $N$-glucosylation on type-1 collagen fibrillogenesis. Biosci. Reproduction, 12: 945-954.

Guthman, F., C. Hohof, H. Fechner, P. Humbert and T. Borchers et al., 1998. Expression of fatty acid binding protein in cells involved in lung spesific lipid metabolism. European J. Biochem., 253: 430-436. DOI: $10.1046 / j .1432-1327.1998 .2530430 . x$

Kang, J., X.S. Dai, T.B. Yu, B. Wen and Z.W. Yang, 2005. Glycogen accumulation in renal tubules, a key morphological change the diabetic rat kidney. Acta Diabetol., 42: 110-116. DOI: $10.1007 / \mathrm{s} 00592-005-0188-9$

Lin, S.S., J.K. Manchester and J.I. Gordon, 2001. Enhanced gluconeogenesis and increased energy storage as hallmarks of aging in Saccharomyces cerevisiae. J. Biol. Chem., 276: 36000-36007. DOI: $10.1074 /$ jbc.M103509200

Manchester, J., A.V. Skurat, P. Roach, S.D. Hauschka and J.C. Jr. Lawrence, 1996. Increased glycogen accumulation in transgenic mice overexpressing glycogen synthase in skeletal muscle. Proc. Natl. Acaded. Sci., USA, 93: 10707-10711.

Mann, D.M.A., P.Q. Sumpter, A. Davies and P.O. Yates, 1987. Glycogen accumulation in the cerebral cortex in Alzheimer disease. Acta Neuropathol., 73: 181-184. DOI: 10.1007/BF00693786

McGowan, S.E and J.M. Snyder, 2004. Development of Alveoli. In: The Lung Development, Aging and the Environment, Harding, R., K.E. Pinkerton and C.G. Plopper (Eds.), Amsterdam. Elsevier Academic Press, ISBN-10: 0080481353, pp: 55-73.

Pinkerton, K.E., F.H.Y. dan Green, 2004. Normal Aging of the Lung. In: The Lung Development, Aging and the Environment, Harding, R., K. Pinkerton, C.G. Plopper (Eds.), Elsevier Academic Press, London, ISBN-10: 0080481353, pp: 213-233.

Rahbar, S. and J.L. Figarola, 2003. Novel inhibitors of advanced glycation endproducts. Biochem. Biophy., 419: 63-79. DOI:10.1016/j.abb.2003.08.009

Ross, R. and M. Post, 2004. Surfactant lipid synthesis and lamellar body formation in glycogen-laden type II cells. Am. J. Physiol. Lung Cell Molecular Physiol., 287: L743-L751. DOI: 10.1152/ajplung.00146.2004

Singh, R., A. Barden, T. Mori and I. Beillin, 2001. Advanced glycation end-products: A review. Diabetol., 44: 129-146. DOI: $10.1007 / \mathrm{s} 001250051591$

Tortora, G.J., 1999. Principles of Human Anatomy. 8th Edn., John Wiley and Sons, ISBN-10: 0471379697 
Verbeke, P., M. Perichon, C. Borot-Laloi, J. Schaeverbeke and H. Bakala, 1997. Accumulation of advanced glycation endproducts in the rat nephron: Link with circulating AGEs During Aging. J. Histochem. Cytochem., 45: 1059-1068. DOI: $10.1177 / 002215549704500804$

Verzijl, N., J. DeGroot, F. Oldehinkel, R.A. Bank and S.R. Thorpe et al., 2000. Age-related accumulation of Maillard reaction products in human articular cartilage collagen. Biochem. J., 350: 381-387.
Zhou, G., C. Li and C. Lu, 2004. Advanced glycation end products induce connective tissue growth factor mediated renal fibrosis predominantly through transforming growth factor $\beta$ independent pathway. Am. J. Pathol., 165: 2033-2043. DOI: $10.1016 / \mathrm{S} 0002-9440(10) 63254-3$ 